\title{
Disminuir la deserción de estudiantes: un estudio etnográfico sobre la tutoría en la Universidad Técnica de Lisboa, Portugal"
}

\author{
Decreasing Students' Dropout: An Ethnographic Study on \\ Tutoring at the Technical University of Lisbon in Portugal
}

\author{
Bernarda Elisa Pupiales Rueda** \\ ORCID: 0000-0002-4311-8418 \\ Universidad del Tolima, Colombia
}

Recibido: 22 de julio de 2019

Revisado: 17 de octubre de 2019

Aceptado: 22 de febrero de 2020

\section{Resumen}

El artículo presenta los resultados parciales de una macro-investigación sobre innovación pedagógica en educación superior, la cual se lleva a cabo con la participación de siete instituciones de educación superior de España, Portugal y Colombia. En este caso se presentan los resultados de la investigación realizada sobre la tutoría para estudiantes, desarrollada en la Universidad Técnica de Lisboa-Portugal, la cual se identifica como una innovación pedagógica orientada a disminuir el nivel de deserción estudiantil. Es una investigación etnográfica a partir de entrevistas en profundidad, observación y análisis de documentos producidos sobre el tema. Se concluye que la tutoría disminuye el riesgo académico y personal al cual los estudiantes se enfrentan en esta etapa de formación. Además, se ofrecen estrategias para lograr la permanencia del estudiante en la universidad. La tutoría igualmente tiene en cuenta la implementación de actividades orientadas al desarrollo profesional del docente.

Palabras clave: Proceso, aprendizaje, deserción, innovación, calidad.

** Autor de correspondencia: Elisa Pupiales Rueda, Universidad Complutense de Madrid, España. Docente Dpto. de Psicopedagogía. Facultad de Ciencias de la Educación. Coordinadora Red de Investigación para el desarrollo de la pedagogía. Universidad del Tolima. Ibagué-Colombia. Correo electrónico: bepupialesr@ut.edu.co 


\section{Abstract}

The article presents the partial results of a macro-research developed on pedagogical innovation in higher education, which was carried out with the participation of 7 institutions of higher education in Spain, Portugal, and Colombia. In the current study, we present the results of the research carried out on student Tutoring developed at the Technical University of Lisbon, Portugal. There, Tutoring was identified as a pedagogical innovation, which is aimed at improving the training process and reducing student dropout. Research was framed in an ethnographic research based on in-depth interviews, nonparticipant observation, and analysis of written documents on the subject. The study concludes that Tutoring manages to identify possible risks that the student faces in the new stage of training at the academic and personal level and offers strategies to respond adequately to the obstacles that arise during formation, achieving the permanence of the student in the university. Regarding the activities planned to improve the teacher's or tutor's performance, there are refresher courses on various topics, aimed at improving the level of pedagogical knowledge.

Keywords: learning, process, desertion, innovation, quality.

\section{Introducción}

El objetivo de la investigación es comprender el proceso que se desarrolla en torno al acompañamiento de los estudiantes por parte de un tutor, con el objetivo de mejorar la posibilidad de culminar la etapa de formación profesional del estudiante de la Universidad Técnica de Lisboa (UTL). Lo anterior, debido a que la deserción es un obstáculo tanto para la institución y la sociedad. A pesar de que el tema ha sido estudiado desde diversas perspectivas, el resultado sobre iniciativas que logren bajar ostensiblemente el nivel de deserción de los estudiantes es aún incipiente (Miranda y Guzmán, 2017; Navarro, Redondo, Contreras, Romero y D'Andreis, 2017; Núñez, Henao y Fajardo, 2012; Quintela, 2013; Velásquez y González 2017).

En este sentido, Colombia ocupa el segundo puesto en el ranking del estudio de abandono universitario de entre los países del cono sur, señalando que desertan del aula universitaria un $42 \%$ de los jóvenes (Banco Mundial, 2015). A nivel mundial, los países con menor tasa de deserción son Japón, Alemania, Francia y Bélgica (Ferreyra, Avitabile, Botero, Haimovich y Urzúa, 2017). En el caso de Europa la deserción se encuentra entre el $20 \%$ y el $55 \%$, según el Espacio Europeo de Educación Superior (EEES) conformado por 47 países (Salmi, 2016).
La deserción tiene diversas causas, a saber, la posible inestabilidad emocional del estudiante, las dificultades económicas, los resultados académicos y la baja calidad de la formación, entre otros (Barrero, Barrero, Borja y Montaño, 2015). La iniciativa de la tutoría en la UTL es una experiencia exitosa que le confiere reconocimiento a nivel europeo. Además, la universidad ha sido invitada por varias instituciones de educación europeas superior para implementar la experiencia de tutoría en otras universidades.

Por otra parte, el tutorado tiene como objetivo mejorar tanto el desempeño de los estudiantes como las competencias del docente a través de un programa de cualificación permanente, estimulando a la vez el desarrollo profesoral. Se ha identificado que a través de la tutoría la institución le ofrece al estudiante herramientas para afrontar el desafío que acarrea la nueva etapa de formación. Así, se identifica que la tutoría impacta a los estudiantes y docentes, y al equipo directivo en la planificación y gestión para garantizar la calidad de la formación (Pupiales, De Castro y Gonçalves, 2019; Zabalza, 2016).

Cabe destacar que la mejora de la calidad de la enseñanza universitaria europea está a cargo de diversas entidades o agencias, las cuales evalúan tanto el proceso de formación como el nivel de 
empleabilidad del estudiante. En el ámbito de la educación superior europea, el aseguramiento de la calidad se lleva a cabo a partir del análisis de los siguientes aspectos: la calidad de los títulos universitarios oficiales, los resultados del aprendizaje y la revisión periódica de las enseñanzas. Lo anterior se logró desde de la cohesión del sistema universitario europeo a partir del Espacio Europeo de Educación Superior (EEES), el cual cohesiona un $98 \%$ de las instituciones. El objetivo del EEEs, fue unificar aspectos que posibilitan mejorar tanto, la calidad de la educación como la empleabilidad de los futuros profesionales. En Europa, el aseguramiento de la calidad se realiza tanto para instituciones y docentes. Entre las agencias europeas orientadas a la evaluación para el aseguramiento de la calidad, se encuentran: ENQA Y ANECA, ENQA, que pertenecen a la Asociación Europea para el Aseguramiento de la Calidad en la Educación Superior y ésta representa a las organizaciones de aseguramiento de la calidad de los estados miembros del EEES.

En este sentido, ENQA es la encargada de diseminar la información, las experiencias y las buenas prácticas en el campo de la educación superior y además vela por la garantía de la calidad en todos los países signatarios del proceso de Bolonia. Hoy en día ENQA agrupa cerca de 50 agencias de evaluación y es una garantía de calidad de Europa. La ENQA (Agencia Nacional de Evaluación de la Calidad y Acreditación) es una organización española encargada de velar por la garantía de la Calidad de la Educación. Dentro de las funciones específicas de ENQA se encuentran: verificar y evaluar las propuestas de los planes de estudio diseñados en consonancia con el EEES. Acredita los programas encargados de la evaluación de los títulos universitarios oficiales antes de su acreditación. Así mismo, tiene un doble objetivo de comprobar si después de su implantación, cada título responde al proyecto por el cual obtuvo la condición de título oficial y evalúa al mismo tiempo los resultados del título. Lo anterior posibilita el reconocimiento mutuo entre los distintos sistemas educativos que conviven en el EEES, al tiempo que ofrecen garantías al estudiante y a la sociedad con respecto a la calidad de las enseñanzas (ENQA, 2018).
Por otro lado, en Colombia es la ley 30 de 1992 la encargada de velar por la calidad de la educación superior “(...), hace referencia a los resultados académicos, a los medios y procesos empleados, a la infraestructura institucional, a las dimensiones cualitativas y cuantitativas del mismo y a las condiciones en que desarrolla cada institución" (Ley 30 de 1992).

En este sentido, los referentes anteriores complementan el propósito del estudio centrado en identificar el proceso desarrollado en la tutoría, como una experiencia de innovación pedagógica orientada a mejorar la calidad de la educación. En este caso centrado en la Universidad Técnica de Lisboa que reporta un menor índice de deserción de estudiantes en los programas de ingeniería entre las universidades europeas.

\section{Método}

La investigación fue de naturaleza etnográfica y se desarrolló a partir de entrevistas en profundidad, de observación del proceso y del análisis de documentos. Las entrevistas posibilitan identificar aspectos de la tutoría que contribuyen a mejorar la calidad de la formación del estudiante y la cualificación del docente. Se identificaron las categorías correspondientes al proceso de acompañamiento y mejora de la calidad de la formación.

\section{Etapas de la investigación}

\section{Identificación de instituciones que desarrollan buenas prácticas para estudiantes $y$ docentes}

El estudio tuvo como objetivo identificar experiencias de innovación en cuanto a formación de docentes y estudiantes. Escogimos seis (6) instituciones de educación superior de Portugal que cumplían con el requerimiento. La UTL cumplió con dos perspectivas, a saber, mejora del desempeño del estudiante mediante el acompañamiento del tutor y la posibilidad de desarrollo profesional del docente a partir de la 
cualificación permanente. La etapa de selección de la institución tuvo una duración de quince días.

\section{Construcción de instrumentos para recolección de información}

Orientados a responder a las preguntas clave para comprender la perspectiva de innovación de la experiencia de tutoría. La etapa se llevó a cabo en tres fases, la primera fue la construcción de las preguntas teniendo en cuenta los objetivos de la investigación, la segunda tuvo que ver con el análisis de las entrevistas para la identificación de las categorías y la última parte la traducción de las entrevistas al portugués.

\section{Aplicación de instrumentos}

Las entrevistas se desarrollaron con base en las preguntas orientadas a ampliar el contexto de estudio y profundizar sobre la experiencia de acompañamiento o tutoría de estudiantes. Se realizaron cerca de cinco (5) visitas a la institución. La etapa tuvo una duración de cuatro semanas.

\section{Resultados y análisis}

El análisis de la investigación se desarrolló teniendo en cuenta el planteamiento del problema, en torno a identificar cómo se desarrolla el acompañamiento del estudiante a través del tutor, orientado a mejorar el logro de los objetivos de aprendizaje, y una mejor adaptación a la nueva etapa de formación, orientados a bajar el nivel de pérdida y deserción del estudiante. Las respuestas obtenidas de las entrevistas se sistematizan y se aplica el programa N-VIVO, en el cual se identifican categorías y testimonios que corresponden a las mismas.

\section{El trabajo de campo}

Se llevó a cabo en la Universidad Técnica de Lisboa de Portugal con una duración aproximada de siete meses. La información se recogió a través de entrevistas, observación de procesos y consulta del material producido en torno al tema.

\section{Participantes}

Las entrevistas se desarrollaron con la participación de los colectivos implicados en el proceso de tutoría, entre los cuales se encontraban: el equipo directivo de la oficina de calidad de la educación, los asesores de docentes y los tutores de los estudiantes. En su momento el tutorado contó con 20 asesores de docentes, 30 tutores de estudiantes y el equipo directivo constituido por dos psicólogas y el coordinador académico de la UTL. Se entrevistó a tres (3) directivos de la oficina de cualificación docente, seis (6) asesores de docentes, y cuatro (4) tutores de estudiantes. A continuación, se describen las actividades desempeñadas por los diversos colectivos que laboran en torno al tutorado:

\section{El equipo directivo}

Evaluaron los resultados y realizaron seguimiento al nivel de deserción de los estudiantes. Además, propusieron perspectivas de mejora y cualificación del docente y del tutor. Las iniciativas de formación respondieron a las dificultades que afronta el docente, tutor y el estudiante en el proceso. La labor se desarrolló a través de la oficina de cualificación encargada de velar por la calidad de la educación en la UTL.

\section{Asesores de docentes}

Asesoraron en cuanto a la utilización de TIC, y para la adecuada toma de decisiones en torno a las estrategias pedagógicas, didácticas y de evaluación. Cabe destacar que fue el docente quien solicitó la asesoría para mejorar la posibilidad pedagógica de un determinado tema. En cuanto al uso de TIC, los pares asesoraron para mejorar la interactividad entre el estudiante y el tema. Además, acompañaron al docente en el proceso de puesta en marcha del uso de TIC.

\section{El tutor de estudiantes}

La labor fue acompañar e identificar con el estudiante los posibles riesgos, tales como la pérdida de cursos, el bajo rendimiento en áreas troncales o la falta de habilidades sociales, evidenciadas como 
obstáculo para que el estudiante lograra un adecuado ambiente de aprendizaje y mejorara las posibilidades de culminar la formación universitaria. Igualmente sugerían al estudiante desarrollar cursos complementarios, orientaron el uso adecuado del tiempo libre, y colaboraron en la construcción de un horario de estudio aplicado especialmente a los cursos que presentaron mayor riesgo de pérdida. Para desempeñarse como tutor el docente recibía formación en temas como coaching, formación pedagógica, didáctica, evaluación, utilización de tIC y otros temas orientados a la actualización en diversos ámbitos (Marques y Pinto, 2014). Se proponen como tutores los docentes que impartían o impartieron algún curso en primero o segundo semestre, por tanto conocían al estudiante asesorado.

\section{Resultados}

La iniciativa de acompañar a través de tutorías dinamizó el proceso de formación del estudiante y de cualificación del tutor y el docente, a continuación, se describe cada ámbito.

\section{Tutoría para estudiantes}

El programa de tutorías se creó en el año 2003, el objetivo fue bajar el nivel de deserción de los estudiantes generado por la pérdida de asignaturas troncales o prerrequisito. El acompañamiento a través de tutoría se llevó a cabo cada quince (15) días, para lo cual existió un cronograma de fechas para su desarrollo.

Una vez analizadas las respuestas, se identificó que la tutoría se presentó como una iniciativa que motivó la participación, desarrolló la autoconfianza y proporcionó al estudiante seguridad en el proceso de formación, lo cual conllevó a la mejora del resultado académico e integral del estudiante.

\section{Cualificación docente}

El docente por su parte se convirtió en el puente entre el estudiante y el conocimiento, en vista de que fue quien diseñó y tomó decisiones en torno al proceso de formación. En este caso se identificó la calidad a través de aspectos como: el desempeño del docente, los recursos tecnológicos y pedagógicos que ofrecía la institución. La cualificación en este caso hizo parte de la política institucional de la UTL y respondió a las necesidades de formación del docente (Aramburuzabala, Hernández Castilla y Ángel-Uribe, 2013; Imbernón, 2011; Pupiales Rueda, 2016; Zabalza, 2016).

A continuación, algunos testimonios sobre cualificación.

"tenemos interés en compartir proyectos formativos con docentes de todas las facultades, además el compartir de esta manera siempre es enriquecedor"

"estamos formándonos para identificar el copyright en los trabajos, ya que este tema es de interés para que no exista el plagio en trabajos que presentan"

"las horas de formación dentro de la universidad son valoradas por los docentes ya que es un espacio que dedicamos para nosotros mismos, por lo general los docentes tenemos una agenda compleja".

\section{Seminario de buenas prácticas pedagógicas.}

Se desarrolló al final de año, los conferencistas se escogieron del grupo de docentes que obtuvieron buen resultado en la evaluación. De la mencionada actividad se obtuvo una relatoría, y de esta se publicaron las memorias (Márquez y Pinto, 2014). Cabe destacar que un $90 \%$ de los docentes, consideran el seminario para motivar la mejora de su desempeño. Sobre el tema los docentes se refieren así;

"aunque no todos acceden a compartir, un $80 \%$ acepta, es una experiencia de crecimiento profesional"

"por lo general los docentes con buen nivel de desempeño, están dispuestos a ofrecerles información adecuada y adecuado acompañamiento del estudiante durante el proceso" 


\section{Tutores formados como Coach}

Se puede afirmar que el tutor humaniza el proceso de adaptación del estudiante en el nuevo ámbito de la educación superior. El curso que dio inicio a dicho ámbito fue de formación como Coach (Sánchez Mirón y Boronat Mundina, 2014). La base subyacente del coaching se apoya en el método socrático, que permite a partir de preguntas la resolución de problemas de forma autónoma, ofreciendo posibilidades para descubrir el potencial y minimizar las dificultades en el proceso. En cuanto a la formación de docentes a través de coaching educativo, los docentes se refieren así en cuanto a la formación como coaching;

"aprendemos a acompañar al estudiante y a identificar los ámbitos en los cuales hay riesgo de pérdida y deserción"

“el estudiante que llega por primera vez al aula de la universidad requiere mayor atención, por tanto la atención es personalizada"

"identificar las fortalezas y limitaciones del estudiante para evitar pérdidas de cursos constituye la forma de acompañarlos como coach"

"aplicar el conocimiento de coach lograr resultados, el estudiante mejora ostensiblemente".

\section{Implementación de tic en el aula}

El docente solicitó asesoría orientada a mejorar el canal de comunicación con el grupo (Amante, Quintas-Mendes, Morgado y Pereira, 2008). En este caso el asesor pedagógico realizó un análisis sobre el tema y luego entre ambos planificaron las actividades complementarias, ejercicios, trabajos y procesos de evaluación, teniendo en cuenta el tema, el número de estudiantes y el nivel de pérdida del curso. Los tutores lo refieren así:

"el docentes asiste a la oficina a buscar ayuda en temas puntuales, se enteran de que existe la posibilidad de asesoría por el comentario que hacen los que han recibido asesoría, por el boca a boca" “el nuevo Espacio de Educación Superior les motiva a procurar clases más dinámicas, como una posibilidad de mejorar la participación"

"la utilización de las TIC, mejora el interés, y la participación del estudiante"

"el uso de la plataforma está siendo un aspecto casi que obligatorio razón por la cual debemos asumir el manejo del espacio virtual”

\section{Asesores en competencias pedagógicas}

Un $50 \%$ de los docentes demandó la asesoría pedagógica. El objetivo del asesor fue lograr que el tema en mención fuera pedagógico, menos extenso y menos complejo para el estudiante. Encontramos además que el $95 \%$, de docentes de ingeniería de la UTL, carecían del conocimiento pedagógico; sin embargo poseían un alto nivel de formación en el saber específico. En definitiva, la labor del asesor consistió en dar respuesta a las inquietudes que surgieron por parte del docente (Pupiales, 2016).

Testimonios de los asesores sobre competencias pedagógicas del docente;

"es ofrecerle posibilidades para presentar el tema para que el contenido, y las actividades sean menos complejas"

"sabemos por experiencia que en algunos cursos de ingeniería el estudiante debe desarrollar capacidad e ingenio"

“ dividir un tema en partes, para que sea más pedagógico"

"ellos manejan con excelencia el ámbito del saber específico, sin embargo carecen de la formación didáctica"

"en ocasiones el docente tiene dificultad para dejarse orientar, existe una dicotomía en este aspecto".

La asesoría, según un docente 
“(...) permite que piense y construya la clase desde la mirada del estudiante, el cual aún desconoce el tema, y hace que el tema se presente de una manera menos compleja, asegurando que el aprendizaje se realice en mejores condiciones, y permite además que el estudiante continúe motivado por el curso" (Docente de matemáticas UTL)

\section{Evaluación, mejora y monitorización de la tutoría}

El tutorado fue monitorizado a través de un sistema de evaluación aplicado en tres etapas; al inicio, en el intermedio y al finalizar el cuatrimestre, para lo cual se aplicaron cuestionarios a estudiantes y tutores, además se analizó el nivel de pérdida y deserción generada en los dos primeros años de ingreso a la educación superior. La calidad de la tutoría se evaluó de forma endógena y exógena, cualitativa y cuantitativamente (Gonçalves, Pile y Patrocínio (2010).

\section{De los talleres complementarios para estudiantes}

Propuestos para mejorar el desempeño. Se identificó que la tendencia del estudiante fue elegir talleres orientados a la adquisición de competencias académicas, tales como; elaboración y presentación de trabajos escritos, presentación en Power-Point, desarrollo de habilidades sociales, fortalecimiento de inteligencia emocional y creatividad. De entre los cursos complementarios el de desarrollo y fortalecimiento de la creatividad obtuvo mayor acogida, la satisfacción del estudiante respecto al curso fue de un $86 \%$. Quizá por ser un tema transversal en los programas de formación de ingenieros, en los cuales el ingenio prima en el proceso.

\section{Conclusiones}

La UTL, dio inicio al programa de tutorías como respuesta al alto índice de pérdida y deserción de los estudiantes de los programas de ingeniería. La tutoría fue una iniciativa que complemento el proceso de formación del estudiante y ofreció herramientas y estrategias para afrontar dicha etapa de formación con mayor seguridad (Pupiales et al., 2019).

La labor del tutor fue prevenir posibles riesgos y pérdidas de cursos, además de potenciar las habilidades del estudiante. La tutoría fue una labor conjunta, entre el tutor y el estudiante. El tutor sugirió y orientó la toma de decisiones del estudiante respecto a temas académicos y psicosociales.

En la uTL la cualificación del docente hace parte de la política institucional, para lo cual cuenta con asesorías para docentes, el acompañamiento se lleva a cabo en el uso de tic y en cuanto al fortalecimiento de competencias pedagógicas.

Como experiencia de buenas prácticas se desarrolló un seminario de fin de curso, en el cual participaron los docentes mejor evaluados, lo cual estimuló al docente para conocer y valorar otras perspectivas pedagógicas orientadas a mejorar la intervención en el aula (Gonçalves et al., 2010).

El éxito del tutorado logró que la UTL se posicione como una de las instituciones de educación superior con menor nivel de deserción respecto a las demás instituciones en Portugal en programas de formación de ingenieros. En este momento se realiza asesoría a instituciones de educación superior interesadas en implementar el programa, tanto a nivel nacional como en otros países de la Unión Europea (Gonçalves, 2011).

La cualificación del docente fortaleció la cultura de la formación permanente y orientó la mejora de la calidad, profundizó en la idea en torno a que la docencia en educación superior requiere permanecer al día sobre cuestiones del saber específico y pedagógico (Imbernon, 2011; Zabalza, 2016).

\section{Debate}

En Colombia y los países del cono sur es necesario que el ámbito de la educación superior adopte medidas orientadas a disminuir la deserción de estudiantes, más aún porque a partir de la etapa de contingencia de la pandemia actual, la educación pasa de ser presencial a formación virtual. Se transforma el medio y por tanto debería transformarse el proceso. 
Colombia es el segundo país de Latinoamérica con el nivel más alto de deserción universitaria. Por tanto, es imperativo que se asuman iniciativas de acompañamiento al estudiante, especialmente en los dos primeros años de formación los cuales se convierten en la etapa más susceptible para la deserción.

En el desarrollo del saber, la relación teoría y práctica se convierte en el eslabón entre el saber hacer y conocer (Delors, 1996). Esta quizá sea una posibilidad para que el docente ofrezca nuevos canales, ámbitos y formas de expresión para que el estudiante demuestre el saber adquirido teniendo en cuenta su estilo de aprendizaje y el tipo de inteligencia que posee, y no únicamente a través de la evaluación basada en pruebas de examen escrito u oral. En el siglo XXI es necesario asumir nuevas formas del proceso e igualmente la evaluación. La teoría de las inteligencias múltiples es una respuesta a la diversidad del aula (Barrero et al., 2015; Gardner, 2001).

Es imprescindible que las instituciones desarrollen políticas que promuevan la profesionalización del docente, las cuales tienen que ver entre otros, con facilitar movilidad académica, ofrecer escenarios para el encuentro y el diálogo entre pares, estimular la aplicabilidad de resultados de investigación pedagógica, en aras de la calidad en la formación (Donoso y Schiefelbein, 2007).

La cualificación permanente del docente se convierte en una inversión que redunda positivamente en la calidad del proceso y es necesario que el Estado, la institución y los docentes asuman el compromiso (Aramburuzabala et al., 2013).

\section{Referencias}

Amante, L., Quintas-Mendes, A., Morgado, L. y Pereira, A. (2008). Novos contextos de Aprendizagem e Educação online. Revista Portuguesa de Pedagogia, 42(3), 99-119. https:// doi.org/10.14195/1647-8614_42-3_6

ENQA (2018). Informe sobre el estado de la evaluación externa de la calidad en las universidades españolas. Recuperado de http://www.aneca.es/Sala-de-prensa/
Noticias/2019/Informe-sobre-el-estado-dela-evaluacion-externa-de-la-calidad-en-lasuniversidades-espanolas

Aramburuzabala, P., Hernández-Castilla, R., Ángel-Uribe, I. (2013). Modelos y tendencias de la formación docente universitaria. Revista Profesorado. Revista de Currículum y Formación del Profesorado, 17, 345-357.

Banco Mundial (2015). página web de Enterprise Surveys.

Barrero, F., Barrero, C., Borja, H. y Montaño, M. (2015). Factores de riesgo asociados a la deserción estudiantil universitaria en programas de pregrado de la Universidad de San Buenaventura, Bogotá (20092013) (Tesis de maestría). Universidad San Buenaventura, Bogotá.

Bejarano, L., Arango, L., Cárdenas, K., Durán, H. y Ortiz, A. (2017). Caso de estudio: caracterización de la deserción estudiantil en la Fundación Universitaria Los Libertadores 2014-1 - 2016-1. Tesis Psicológica, 12, 138-160.

Consejo Nacional de Acreditación CNA. (S.F.). La calidad en la acreditación institucional. Disponible en: www.cna.gov.co/1741/article-190811.html.

Congreso de la República de Colombia (28 de diciembre 1992). Ley 30 de 1992. Bogotá, Colombia.

Criterios y directrices para el aseguramiento de la Calidad en el Espacio Europeo de Educación Superior. (ESG). Texto original aprobado por los ministros de Educación de los estados signatarios en la Conferencia Ministerial de Ereván el 14 y 15 de mayo de 2015. Recuperado en: https://enqa.eu/indirme/esg/ESG\%20in\%20 Spanish_by\%20ENQA.pdf

Delors, J. (1996). La educación encierra un tesoro. Madrid: Santillana/Unesco.

Donoso, S. y Schiefelbein, E. (2007). Análisis de los modelos explicativos de retención de estudiantes en la universidad: una visión desde 
la desigualdad social. Estudios pedagógicos. Valdivia, 33, 7-27.

Ferreyra, M., Avitabile, C., Botero J., Haimovich, P F. y Urzúa S. (2017). Momento decisivo: la educación superior en América Latina $y$ el Caribe. Resumen. Washington, D.C.: Banco Mundial.

Gardner, H. (1995). Inteligencias múltiples. La teoría en la práctica. Barcelona: Paidós.

Gonçalves, I. (s/f). A Psicologia no ensino superior: intervenções clínicas e não clínicas. Gabinete de Apoio ao Tutorado, Instituto Superior Técnico, Universidade Técnica de Lisboa. Recuperado en: http://tutorado.tecnico.ulisboa.pt/files/sites/40/A-PSICOLOGIA-NO-ENSINO-SUPERIOR.pdf

Gonçalves, I. (2011) Programa de Tutorado: Orgânica, Missão e Objectivos in Programa de Monitorização e Tutorado: Oito Anos a Promover a Integração e o Sucesso Académico no IST Lisboa: IST Press.

Gonçalves., I. L. A. y Maura, G. (2014). Tutoring Support Office: Increasing Teacher - Student contact in Higher Education, Promoting a shift from Teaching to Learning?. 9th European Quality Assurance. University of Barcelona, Spain. Recuperado en: http://www.eua.be/ Libraries/eqaf-2014/la_2_Goncalves_Lucas_ Moura_Sereno.pdf?sfvrsn=0

Gonçalves., I y Pile., M. (s/f.) Programa de monitorização-tutorado. NAP - Núcleo de Aconselhamento Psicológico e GEP - Gabinete de Estudos e Planeamento. Recuperado en: http://users.isr.ist.utl.pt/ msilveira/ objectivos.pdf

Gonçalves, I. L. A., Pile, M. y Patrocínio, C. (2010). Internal Quality Assurance Processes at IST towards a manual of best practices in teaching, paper presented at the Learning and Teaching in Higher Education Seminar (Évora,
April) - Recuperado de: https://dspace.ist.utl. pt/bitstream/2295/645275/1/Learning\%20y\%20 Teaching\%20VFinal[1]

Imbernón, F. (2011). La formación pedagógica del docente universitario. Revista Educação (UFSM), 36, 387-395.

Londoño, L. (2013). Factores de riesgo presentes en la deserción estudiantil en la Corporación Universitaria Lasallista. Revista Virtual Universidad Católica del Norte, 38, 183 - 194.

Marques, J. y Pinto, P. R. (2014). Formação Pedagógica de Professores do Ensino Superior A Experiência na Universidade Nova de Lisboa. Revista Portuguesa de Pedagogia, 129-149.

Ministerio de Educación Nacional, MEN (2009). Deserción estudiantil en la Educación Superior: Metodología de seguimiento, diagnóstico y elementos para su prevención. Bogotá: Ministerio de Educación Nacional.

Miranda, M. A. y Guzmán, J. (2017). Análisis de la deserción de estudiantes universitarios usando técnicas de minería de datos. Formación Universitaria, 10, 61-68.

Navarro, C. N. E., Redondo, B. O. E., Contreras, J., Romero D. C. y D'Andreis Z. A. C. (2017). Permanencia y deserción versus autoeficacia de estudiantes universitarios: un desafío de la calidad educativa. Revista Lasallista de Investigación, 14, 198-206.

Núñez, M., Henao, A. y Fajardo, E. (2012). “La deserción: un fenómeno social". Vivencias de los estudiantes del programa de enfermería de la universidad del Tolima [Tesis de Maestría].

Observatorio de educación superior de Medellín (2017). Boletín. Deserción en la educación superior. Recuperado en: http://www.sapiencia. gov.co/wp-content/uploads/2017/07/BOLETIN_ ODES_DESERCION_EN_LA_EDUCACION_ SUPERIOR.pdf 
Pupiales, R. E., De Castro, A. y Gonçalves, S. (2019). Innovación en Educación Superior: Un informe iberoamericano. Centro de innovación y estudio de la pedagogía en educación superior. Instituto Politécnico de Coimbra. Portugal.

Pupiales, B (2016). Research in teacher training and learning discipline in the context of the university classroom. Tendencies in the training of university teachers: An ethnographic study in six portuguese higher education institutions. Universidad de la Laguna. España.

Pupiales Rueda, E. (2016). Research in Teacher Training and Learning Discipline in the context of the university classroom. Tendencies in the Training of University Teachers: An Ethnographic Study in Six Portuguese Higher Education Institutions, by Bernarda Elisa Rueda Pupiales. Study of case in depth. Universidad de la Laguna. España.

Pupiales Rueda, E y de Fonseca Tinoca, L. (2014). La Formación universitaria en Iberoamérica desde lo virtual, como entorno para la calidad pedagógica en la educación superior. Revista TENDENCIAS, 15, 268- 286.

Quintela D. E. (2013). Deserción universitaria, una aproximación sociológica al proceso de toma de decisiones de los estudiantes. Sociedad Hoy, 24, 83-106.

Sánchez Mirón, B, y Boronat Mundina, J. (2014). Coaching Educativo: modelo para el desarrollo de competencias intra e interpersonales. Educación XX1, 17(1), 221-242.

Salmi, J. (2016). Combatir la deserción en la educación superior: lecciones a partir de la experiencia internacional.

Velázquez, N. Y. y González, M. (2017). Factores asociados a la permanencia de estudiantes universitarios: caso UAMM-UAT. Revista de la Educación Superior, XLVI (184), 117-138.

Zabalza, M. (2016). Ser profesor universitario hoy. La cuestión universitaria, 5, 68-80. 


\section{Anexos}

\section{Guião de entrevista a Acompanhantes o Asessores de Docentes universitarios.}

La entrevista tem como objectivo analisar na perspectiva a partir da qual tem sido realizado o acompanhamento dos tutores nel projecto de tutorado da UTL.

Desde já se agradece a sua participação e colaboração.

Instituição:

\section{Departamento:}

Idade: até $35 \_$entre 36 e $45 \_$entre 46 e 55 entre 56 e 65

Formação: Mestrado___Doutoramento___ Outra

(Qual)

Categoria Docente: Assistente _- Professor Auxiliar ___ Professor Associado __ Professor Catedrático

Experiencia nel tutorado: até três anos_- entre 4 e 7 anos__ entre 8 e 12 anos _- Mais de 12 anos

La formaçao universitaria requiere altos niveles de qualidade, por cuanto é ela na responsable da formaçao dos profesionais em os diversos ámbitos do saber, em esta medida o desenvolvimento dos docentes universitarios é o primeir peldaño em importança para la melhora de a qualidade de ensino superior.

1. Gosta de sua profissão? Sim __ Não __ por qué?

2. En qué ámbito da acomphanamento percibe que os estudantes teim maior dificuldade em em no tecnológico __ o Pedagogico ambos por igual
3. Depois de o processo de Bolónia e mayor a motivaçao por a melhor e ele desenvolvimento nel estudante?:

4. Como é a motivação dos estudantes pelo acompanhamento do tutor? Alta__Media___Media baja Baja

5. Há quanto tempo se implementó o acomphanamento de tutores na UTL?

6. Qual é e perfil dos docentes que aceptam sert tutores?

7. Quaís sao as estrategias máis requeridas pelo estudantes para a melhora de su desempenho como estudante:?

8. Como se determina o tipo de estrategia o apoio?, Se faz según edad del estudante? según temáticas na quales tenha dificuldade?

9. Depois da assesoria se realiza algum tipo de seguimento del estudante y el resultado nel aula?

10. Quiem proponha fazer parte das asssorias de tutores, os docentes o estudantes?

11. Qual é a época do ano, que já maior demanda de asesoria e de acomphanamento?

12. Faz-se algum análisis, em quanto a os resultados de melhora do processo nel aula comentados pelo estudantes?

13. O acompanhamento tein-se em conta para a futura contrataçao dele docente em na instituição?

14. Qual é a duração em horas de um processo do acompanhamento e tutoria? 
15. Quantas pessoas participam nel equipa de tutorado de docentes? _- Quais e o papel de cada uma?:

16. Anterior a esta, que formação o asessoria recebiam os docentes?

17. Em qué área o ámbito o acompanhamento e mais complejo de desenvolver?
18. Entre quantas horas semanais dedica em casa ao trabalho de disenho de asessoria o tutor? (preparar disenho, ler, construir materiais, planificar actividades para os docentes)?

Menos de 10 horas__Entre 11 e 15 horas_Entre 16 e 20 __ Mais de 20 horas

Obrigada por sua colaboração 